\title{
Design of Transformer Temperature Rising Monitoring and Alarming System Based on Zigbee Technology
}

\author{
Liang Wenjie \\ College of Automotive Engineering,Weifang University of Science and Technology \\ Shouguang , 262700,China \\ email: 274729523@qq.com
}

Keywords: Transformer temperature, Zigbee, monitoring and alarm

\begin{abstract}
For the shortcomings of current transformer monitoring alarm systems exist in data transmission, designing transformer monitoring alarm system based on ZigBee technology.Systems with TI's CC2430 ZigBee module as the core, using a precision chip DS18B20 as digital temperature sensor for designing temperature monitoring terminal and realize the transformer temperature monitoring system wireless basically. The system can save a lot of costs, reduce the complexity of construction, and has broad application prospects.
\end{abstract}

\section{Introduction}

Transformer transformer temperature rise means working a certain time, because of their power loss and fever, causing a higher temperature than the value of each part of the interior outside temperature.Transformer temperature rise to characterize the insulating function of the transformer, the transformer is one of the important indicators of technical performance is directly related to the safety of the transformer, reliability and service life.Transformers national standard for transformer temperature rise and temperature tests are clearly defined.The purpose of the test is to verify the transformer temperature rise under the provisions of the state of the transformer windings, core and transformer oils and to find housing, fuel tanks, structural parts, wire and casing and connections as well as lead the tap without local overheating;determining the transformer at rated working condition and super hot plate load status and related parameters compliance testing requirements of the relevant standards and technical protocols temperature rise test on the quality and reliability of the transformer has an important role.

\section{The Introduction of ZigBee Technology}

Zigbee technology is a two-way wireless communication technology, generally applicable to a shorter distance, less complex, less power consumption, low-cost data transmission information.Reference herein to monitor the temperature of the transformer short-range wireless transmission terminal basically a low-power, short distance, and data transmission between the transmission rate is low intelligence module, the data they transport cyclical, low reactivity intermittent time data type.igBee communication technology has low power consumption, high data transmission reliability, the advantages of a short delay.

The greenhouse monitoring and control system for each collection terminals are compact, low-cost devices. Wireless networking between them does not require high bandwidth, low latency requires only low power consumption can be, so that each terminal of the communication module may communicate data through Zigbee network.

\section{Institutions Optimization Design}

Wireless meter reading system network architecture shown in Fig.1, the overall structure is divided into five sections:Zigbee network, the smallest single-chip systems,storage section, the alarm portion and temperature acquisition section.System mainly works: temperature acquisition 
module temperature data collected after the transmission of the transformer to the microprocessor and data storage temperature, the microprocessor data analysis to determine if the transformer temperature within the collection is not permissible range, the micro-processing alarm module starts immediately and send an alarm signal first bit machine via Zigbee network.

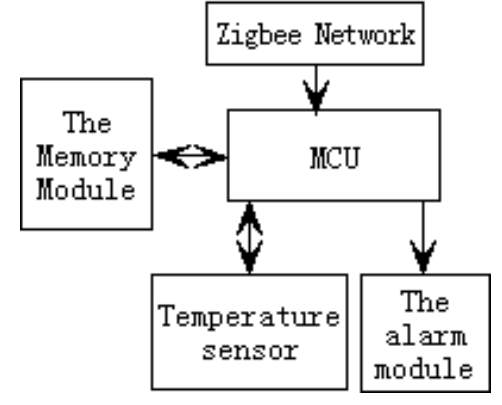

Fig.1 The picture of overall design

\section{Hardware Design}

\section{Zigbee Network}

Zigbee module and microcontroller connection principle shown in Fig.2. Zigbee module selection ZIC2410 chip, MCU serial connection Zigbee module, the data transfer process, the baud rate can be carried out within 1200bps-115200bps range selection, the module has a larger output power, maximum up to $8 \mathrm{dBm}$, and ideally By having a visual transmission distance of 500 meters.

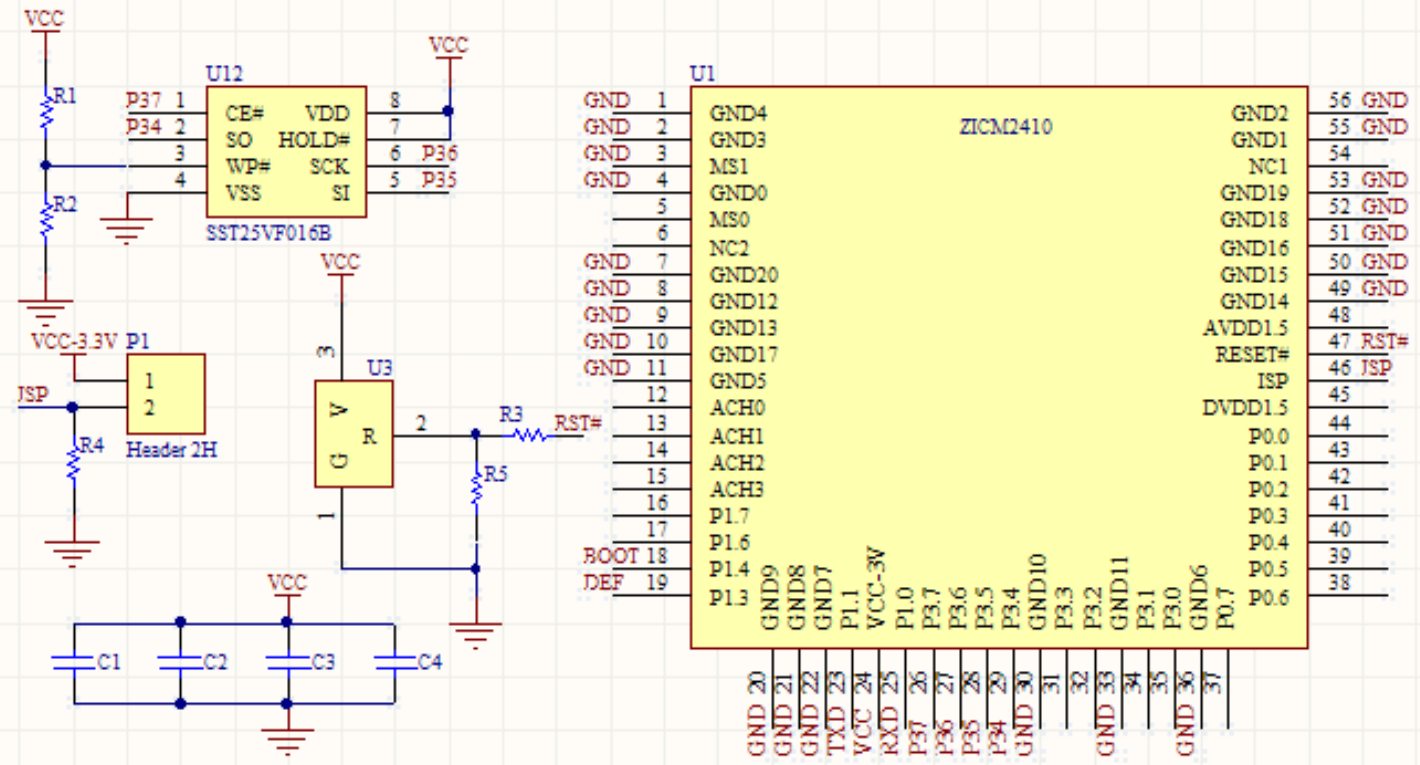

Fig.2 Zigbee network diagram

\section{Microcontrollers}

STC15F2K60S2 SCM is a high speed, high reliability, interference of a new micro-controller chip, high-speed, high reliability. High stability, with strong anti-jamming capability, ultra-low-power dual-independent high-speed asynchronous serial interface, the system is easy to expand.

\section{Storage Section}

In this paper, a memory module selection FM24C64 based ferroelectric memory chip technology, FM24C64 is 64kb serial ferroelectric memory chip RAMTRON provided, FRAM (Ferroelectric Random Access Memory) has a non-volatile, using the SOIC-8 package form. Use I2C bus interface to read and write data between the microcontroller implementation and operation of the chip WP pin main achievement of the chip write protection, this pin is low, you can write the chip of the chip; HIGH , then the part of the storage area of the chip as a hardware write protection, he incurs the protected area data write operation can not be performed, but only read the data. 
The design of the master host microcontroller STC15F2K60S2 mouth and SCL P3.1 P3.0 pin and FM24C64 memory and SDA pins. In order to ensure the normal data read between the microcontroller STC15F2K60S2 and FM24C64, must be made in the work program the microcontroller STC15F2K60S2 appropriate timing to produce a serial data, and taking into account the make SCL, SDA remain high in the serial data line pullup resistor on the access. Principle memory module diagram design shown in Fig.3.

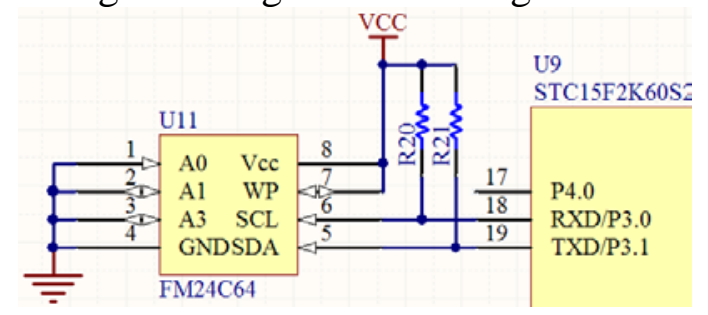

Fig.3 Storage Module Schematic

\section{Alarm Section}

The device is designed alarm circuit, in addition to sound, light alarm, the acquisition module also sends alarm messages via Zigbee network up crew. Where sound, light alarm circuit diagram shown in Fig.4.

\section{Temperature Acquisition}

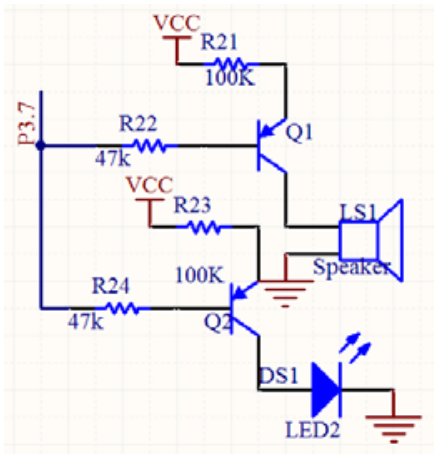

Fig.4 Alarm circuit

TMP102 digital temperature sensor produced by TI, is one with dual bus, special low-power characteristics of the chip,the chip uses ultra-small SOT563 package, ideal for small space in this design of the PCB board. When measuring the temperature, the effective range is $-40 \mathrm{C}-+125 \mathrm{C}$ and has a very high accuracy, the error value is only $0.0625 \mathrm{C}$. Low power consumption of the chip significantly lower maximum current work status $10 \mathrm{uA}$, shutdown mode only $1 \mathrm{uA}$, this design is a terminal monitoring module the best choice when using battery power, the work of the rated voltage of the chip is $1.4 \mathrm{~V}-3.6 \mathrm{~V}$.

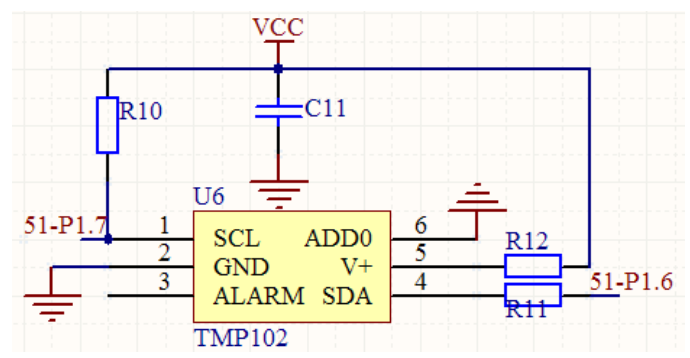

Fig.5 Temperature acquisition circuit schematics

\section{Design of System Software}

Using C51 language for software program, software design mainly includes transformer temperature networking program for the collection, storage and Zigbee networks as well as real-time monitoring and alarm greenhouse design.Prepare the initialization process and the entire system contains the connection Zigbee network ready,after the initialization is complete,the system 
began collecting transformer temperature, and then save. When the temperature is not within the scope of the transformer pre-set, the system begins to alarm, and send alarm messages via Zigbee network up crew to the wait staff tuned transformer. The main flow of the monitoring system shown in Fig.6.

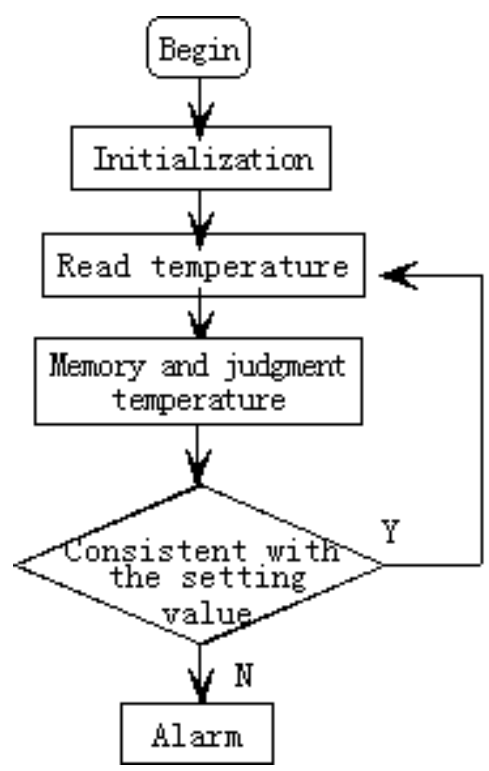

Fig.6 Overall flowchart software

\section{Conclusion}

Previously, there transformer design method for temperature measurement and control system is too many, but usually can not be achieved purely electronic devices or remote monitoring, the paper-based ultra-low power microcontroller on the Zigbee communication technologies combine to make the remote monitoring of the transformer a major breakthrough, thereby reducing the overall cost of the system. According to the design of the system, can be achieved on-line monitoring of transformer temperature data for the grasp of the transformer running state has great significance, I believe in the future of this system will be a great promotion. The system can meet the power transformer operating system security requirements, and low power consumption, easy to use, has a very broad application prospects.

\section{References}

[1]STC15F2K60S2 single chip manual,WWW.STCMCU.COM.

[2]Zhang Yingxin. SCM beginner tutorial:The concept of SCM(The second edition )[M]. Beijing: Beihang University press ,2006.211-229

[3]Wang Gangshi.Computer Control on Multimeter Measurement Instrument Based on RS232 in LabVIEW. Proceedings of the Second International Symposium on Test Automation \& Instrumentation (Vol.4), 2008,11(1):270-274

[4]Li Daohua Etc. . Analysis and Design of the Sensor Circuit[M] . Wuhan: Wuhan University press , 2000.

[5]Zhou Jianyu - Wireless Temperature Acquisition System Based on Zigbee Technology[D] . Wuhan: Hubei university of Technology. 2011.12. 